TW4-29-01-2015

Running head: CROSS-CULTURAL ANALYSIS OF CAREER DECISION MAKING

\title{
Career Decision-Making Profiles and Career Decision-Making Difficulties: \\ A Cross-Cultural Comparison among US, Israeli, and Chinese Samples
}

\author{
Tirza Willner, Itamar Gati and Yanjun Guan \\ Hebrew University of Jerusalem University of Surrey
}

January 2015

Tirza Willner

Dept. of Psychology

The Hebrew University

Jerusalem 91905, ISRAEL

Tel: +972-528-682512 Fax: +972-2-5882084, e-mail: tirzawillner@yahoo.com

\section{Corresponding Author:}

Prof. Itamar Gati

Dept. of Psychology

The Hebrew University

Jerusalem 91905, ISRAEL

Tel: +972-2-5882170, Fax: +972-2-5882084, e-mail: itamar.gati@huji.ac.il

Prof. Yanjun Guan,

Faculty of Business, Economics, and Law

University of Surrey, Guildford, Surrey GU2 7XH, United Kingdom

Tel: +44-1483689241, Fax: +44-1483686346, email: y.guan@surrey.ac.uk

\section{Authors Note}

We thank Nitzan Barak, Eitan Bocian, Naomi Goldblum, Shahar Hechtlinger, Valentina Izrailevitch, Nimrod Levin, Yuliya Lipshits-Braziler, Maya Perez, Dana Vertsberger, and Nitzan Yeshanov for their comments on previous versions of this paper. Part of the present research was presented as an E-poster at the $28^{\text {th }}$ ICAP Convention in Paris, France. This research was supported in part by the Samuel and Esther Melton Chair of the second author and National Natural Science Foundation of China (NSFC, Project ID: 71102107) of the third author. Correspondence concerning this article should be addressed to Itamar Gati, Department of Psychology, The Hebrew University, Jerusalem 91905, ISRAEL. E-mail: itamar.gati@huji.ac.il. 


\title{
Career Decision-Making Profiles and Career Decision-Making Difficulties: A Cross-Cultural Comparison among US, Israeli, and Chinese Samples
}

\begin{abstract}
Assessing individuals' career decision-making difficulties and career decision-making profile (style) allows counselors to help them make better career decisions. The present study focused on the associations between the Career Decision-Making Profiles (CDMP) questionnaire and the Career Decision-making Difficulties Questionnaire $(C D D Q)$ in four large samples: American Adults $(N=601)$, Israeli young adults $(N=623)$, American students $(N=$ 915), and Chinese students $(N=929)$. The pattern of associations between the $12 C D M P$ dimensions and the $10 C D D Q$ scales was generally replicated across the four samples, and indicated that certain $C D M P$ dimensions were associated with career decision-making difficulties. Additionally, in all four samples, the higher an individual's career decision-making adaptability, as derived from the CDMP, the fewer difficulties he or she encountered prior to and during the career decision-making process. However, a few unique patterns of associations between the $C D M P$ and the $C D D Q$ emerged in some samples.
\end{abstract}

Highlights (MAX 85 including spaces):

- Associations between career decision-making profiles and difficulties were tested.

- The associations were tested in 4 samples from the US, Israel, and China $(\mathrm{N}=3068)$.

- $\quad$ The pattern of associations was generally similar across the samples.

- Associations in the Chinese sample were less similar to those in the other samples.

- $\quad$ Career decision-making adaptability was associated with fewer difficulties.

Keywords: Career indecision, career decision-making style, CDDQ, CDMP, career decisionmaking profiles, career decision-making difficulties, cross-cultural 


\section{Career Decision-Making Profiles and Career Decision-Making Difficulties: A Cross-Cultural Comparison among US, Israeli, and Chinese Samples}

\section{January 2015}

Individuals face many important decisions during their lifetime, where choosing a college major is among the most important decisions young adults have to make. The outcome of choosing a less then optimal career path is costly, with significant financial and psychological consequences for the individual's life and indirect social consequences for the society as a whole (Gillie \& Gillie-Isenhour, 2003). The importance of the decision may contribute to the stress that is often caused by the need to make such an important decision (Lipshits-Braziler, Gati, \& Tatar, 2015) and this stress can increase the difficulties encountered during the career decision-making process. Additionally, the numerous career paths and the very large number of career possibilities in the $21^{\text {st }}$ century can make this difficult process even harder.

To understand the sources of career decision-making difficulties and describe the factors associated with more difficulties, previous research has evaluated the relationship between career decision-making difficulties and various individual characteristics. This includes locus of control (Lease, 2004), self-efficacy (Sidiropoulou-Dimakakou, Mylonas, Argyropoulou, \& Tampouri, 2012), dysfunctional thinking (Kleiman et al., 2004; Sidiropoulou-Dimakakou et al., 2012), and personality traits as measured by the Big Five model of personality (Di Fabio \& Palazzeschi, 2009). It has been found that a more external locus of control predicted more career decisionmaking difficulties (Lease, 2004), lower levels of self-efficacy were associated with both more dysfunctional thoughts and more career decision-making difficulties (Sidiropoulou-Dimakakou et al., 2012), and, finally, lower levels of extroversion and higher levels of neuroticism were associated with more career decision-making difficulties (Di Fabio \& Palazzeschi, 2009). Following this line of research, the present study focused on the associations between the way 
individuals make career decisions, as measured by the Career Decision-Making Profiles $(C D M P)$ questionnaire, and their career decision-making difficulties, as measured by the $C D D Q$ (Gati, Krausz, \& Osipow, 1996), in four large samples of young adults.

\section{Career Decision-Making Difficulties}

Career indecision is among the most salient issues in vocational psychology (Osipow, 1999). In response to Tinsley's (1992) call for more theoretically-based research on career indecision, Gati et al. (1996) developed the taxonomy of career decision-making difficulties and the Career Decision-making Difficulties Questionnaire $(C D D Q)$, which is based on it, expanding previous theoretical and empirical research (e.g., Campbell \& Cellini, 1981; Miller, 1971). The $C D D Q$ stems from a decision-theory viewpoint (Gati, 1986; Katz, 1966; Pitz \& Harren, 1980) and assesses deliberating individuals' career decision-making difficulties in terms of a multidimensional taxonomy (Gati et al., 1996). The taxonomy consists of three major difficulty clusters, divided into 10 specific difficulty categories. The first cluster, Lack of Readiness, includes three difficulty categories that may arise prior to the career decision-making process: (1) lack of motivation, (2) general indecisiveness, and (3) dysfunctional beliefs. The second and third clusters include difficulty categories that may arise during the career decision-making process. The second major cluster includes four categories, involving Lack of Information about: (4) the career decision-making process, (5) the self, (6) occupations, and (7) ways of obtaining additional information. The third major cluster includes three categories, involving Inconsistent Information due to (8) unreliable information, (9) internal conflicts, and (10) external conflicts. The validity of both the taxonomy and the questionnaire has been empirically tested and supported (Creed \& Yin, 2006; Gati et al., 1996; Gati, Osipow, Krausz, \& Saka, 2000; Gati \& Saka, 2001a, 2001b; Kelly \& Lee, 2002; Lancaster, Rudolph, Perkins, \& Patten, 1999; Mau, 2001; Osipow \& Gati, 1998; Tien, 2005; Vahedi, Farrokhi, Mahdavi, \& Moradi, 2012). 


\section{Career Decision-Making Profiles}

The importance of the way individuals approach the challenge of making a career decision was recognized some time ago (Harren, 1979). Expanding and elaborating on Harren's typology, Gati, Landman, Davidovitch, Asulin-Peretz, and Gadassi (2010) developed the Career Decision-Making Profiles (CDMP) questionnaire, aimed at characterizing the way individuals make career decisions with a multidimensional approach. Like the $C D D Q$, the $C D M P$ focuses on individual characteristics involving the process of career decision-making and not the content of career decision-making (i.e., interests, values, abilities; Gati \& Levin, 2012). Previous theoretical research defined individual differences in career decision-making in terms of a few types, according to the individual's most dominant trait or characteristic (e.g., rational, intuitive, dependent; Harren, 1979). Relying on the individual's dominant characteristic way of making career decisions disregards important individual differences in additional aspects, even if they are less salient. Accordingly, the $C D M P$ views individual differences in career decision-making differently: it provides a multidimensional mapping of the individual's way of approaching career decision-making. Previous research found the $C D M P$ beneficial in evaluating individual differences in making career decisions, compared to viewing individual differences in terms of a single dominant characteristic (e.g. career decision-making styles; Gati, Gadassi, \& MashiahCohen, 2012). Specifically, the CDMP makes it possible to assess individual differences while simultaneously considering the following 12 dimensions (Gati et al., 2010; Gati \& Levin, 2012): Information gathering (comprehensive vs. minimal), Information processing (analytic vs.

holistic), Locus of control (internal vs. external), Effort invested (much vs. little), Procrastination (high vs. low), Speed of making the final decision (fast vs. slow), Consulting with others (high vs. low), Dependence on others (high vs. low), Desire to please others (high vs. low), Aspiration for an ideal occupation (high vs. low), Willingness to compromise (high vs. low), and Using 
intuition (high vs. low).

For six of the $12 C D M P$ dimensions, one of the poles is more adaptive for career decision making (i.e., facilitates the career decision-making process more) than the other: comprehensive Information gathering, a more internal Locus of control, less Procrastination, greater Speed of making the final decision, less Dependence on others, and less Desire to please others (Gadassi, Gati, \& Dayan, 2012; Gadassi, Gati, \& Wagman-Rolnick, 2013). Based on these six dimensions, Gati and Levin (2012) defined a measure of Career Decision-Making Adaptability (CDA). A higher $C D A$ score represents a more adaptive career decision-making profile, and hence a better ability to make a decision. In the present research, we hypothesized that greater adaptability would be associated with fewer career decision-making difficulties.

\section{The Associations between Career Decision-Making Profiles and Difficulties}

The discussion on both career decision-making difficulties and career decision-making profiles stems from a perspective that focuses on the career decision-making process rather than its outcome - the degree of fit between individuals and their work environment. The $C D M P$ describes the way individuals approach career decisions, thus focusing on the question "How do individuals make career decisions?" The $C D D Q$, in contrast, assesses the different types of difficulties that an individual might encounter before or during the career decision-making process. Accordingly, it can be said that the $C D D Q$ focuses on the question "What prevents the individual from making a career decision?" The assumption underlying the present study is that the way individuals make their decision affects the types of difficulties they encounter during the process.

The $C D A$, which can be derived from the six adaptive $C D M P$ dimensions, and the $C D D Q$ can be viewed as representing different perspectives on the process. Whereas career decisionmaking difficulties can be seen as a construct focusing on the factors that prevent the individual 
from advancing in making a career decision (Gati et al., 1996), career decision-making adaptability refers to the individual's ability to proceed and make the decision without any unnecessary delays after taking the relevant information into consideration (Gadassi et al., 2012). Thus, career decision-making adaptability refers to a specific aspect of Savickas' broader construct of career adaptability - "the attitudes, competencies, and behaviors that individuals use in fitting themselves to work that suits them" (Savickas, 2005, p. 45). Therefore, we claim that the more adaptive the individual's career decision-making profile is, the fewer difficulties he or she will encounter during the process.

\section{Cultural Differences in Career Decision-Making}

Individuals in different countries are likely to make their career decisions differently. While some of these differences are based on the system of higher education (such as choosing a major before applying to college rather than after one's first or second year; Clark, 1986), other differences are more contextual, depending on the culture and its different expectations of the individual (Mau, 2004). However, even though individuals from different cultures make career decisions differently (Mau, 2004), most of the research on career decision-making has been conducted in western cultures, so it is important to expand the discussion and the empirical research on career decision-making in general, and career decision-making assessment in particular, to different cultural groups (Blustein \& Ellis, 2000).

One of the prominent ways of distinguishing among cultures is based on the individualism-collectivism dimension. Individualistic cultures, which include most Western countries (Mau, 2004) usually, promote independence and individual autonomy (Hui \& Villareal, 1989; Mau, 2004). Whereas collectivistic cultures, which include most Asian, Latin American, and African countries (Mau, 2004), usually promote interdependence, the society's role in the individual's life and decisions, shared resources and outcomes, social conformity, and 
the importance of family expectations (Hui \& Triandis, 1986; Mau, 2004). Based on this distinction, several cross-cultural comparisons of career decision-making have been made. Mau (2000), for instance, found that Taiwanese students were more likely than American students to embrace a dependent decision-making style and less likely to embrace an intuitive one.

Additionally, studies have translated, adapted, and tested the validity of the current assessment tools in additional cultures (CDMP; Tian et al., 2014; $C D D Q$; Creed \& Yin, 2006; Mau, 2001) and compared the different patterns of responses among various cultures (CDMP; Guan et al., 2015; CDDQ; Mau, 2004). For example, Guan and his colleagues (2015) tested responses to the $C D M P$ as well as other assessment instruments in an American and a Chinese sample of undergraduate students, and found that Chinese students scored significantly higher than American students on the CDMP dimensions of Desire to please others, Dependence on others, Consulting with others, Willingness to compromise, and Procrastination. However, they scored lower in Effort invested, Aspiration for an ideal occupation, and internal Locus of control. Mau (2004) evaluated differences in $C D D Q$ responses among different ethnicities in the US, and found that Asian-Americans had greater difficulties then Caucasian-Americans, in both highschool and college samples. Another study by Mau (2001) found that Taiwanese undergraduate students experienced more career decision-making difficulties than American students. Furthermore, this study showed that while the American students' career indecision was associated with difficulties involving Lack of Readiness, for the Taiwanese students it was associated with difficulties involving Lack of Information.

Career development is particularly challenging in Israel. Due to the mandatory military service after high school, high-school counselors devote minimal, if any, attention to issues of career development, and do not directly encourage students to think about their future career. In contrast, the young adults' military service, which is often followed by temporary work and then 
a backpack trip abroad of several months or a year, promotes self-exploration. Thus, Israeli young adults typically begin the process of formulating their career path when they are not in an educational or vocational setting, with no easily accessible source of support, except for their family, friends, and the Internet (Vertsberger \& Gati, 2015). The fact that Israeli young adults have to declare a major on applying to college is an additional factor that distinguishes the Israeli sample from the others.

Thus, in this study we hypothesized that individuals from different cultures will differ in both the relative salience of their career decision-making difficulties and their typical career decision-making profiles. First, there are cultural differences in the timing of deciding which college to attend and then which major to study. Second, generally speaking, the US is an individualistic society, Chinese is collectivistic, and Israel is somewhere in between; thus, we hypothesized that the way one makes decisions has different implications in each culture. To sum up, we explored whether the pattern of associations between the $C D M P$ and $C D D Q$ varies among the cultures.

\section{The Present Study}

The aim of the present study was to evaluate the association between deliberating individuals' career decision-making difficulties and their career decision-making profiles. Using the Career Decision-Making Profiles (CDMP) questionnaire, we assessed the association between the $12 C D M P$ dimensions, on the one hand, and the three major difficulty clusters, and the overall score derived from the Career Decision-making Difficulties Questionnaire (CDDQ), on the other hand. We also tested the association between the $C D A$ index of the $C D M P$ and the dimensions of the $C D D Q$. Illuminating the relations between the $C D M P$ and the $C D D Q$ in culturally different samples is important for understanding whether the associations are universal or culturally dependent. 
Pervious research found the $C D M P$ to be highly stable over a one-year period, with a median within-participant correlation of .81 across the 12 dimensions (Gati \& Levin, 2012), whereas research on the $C D D Q$ found that intervention reduces career decision-making difficulties (Fouad, Cotter, \& Kantamneni, 2009; Perdrix, Stauffer, Masdonati, Massoudi, \& Rossier, 2012), indicating that this construct is more situational. Therefore, in the current study we hypothesized that the $C D M P$ scores would predict the $C D D Q$ scores, and not the other way around.

Furthermore, using the four large samples of young adults, we tested the hypothesis that the six dimensions of the $C D M P$ that define the $C D A$ would be associated with career decisionmaking difficulties. Specifically, we hypothesized that high Procrastination, high Dependence on others, and high Desire to please others would be positively correlated with career decisionmaking difficulties, whereas comprehensive Information gathering, an internal Locus of control, and a faster Speed of making the final decision would be negatively correlated with these difficulties. We also predicted that the overall $C D A$ score would be negatively correlated with the individuals' career decision-making difficulties as measured by the $C D D Q$. Additionally, we tested whether the strength of the associations between the $C D M P$ scores and $C D D Q$ scores would be different among the culturally different samples.

\section{Method}

\section{Participants}

The participants were obtained from four groups. The data from the first and second groups were collected from people who entered the English $(\mathrm{N}=601)$ or the Hebrew $(\mathrm{N}=623)$ versions of the "Future Directions" website (www.cddq.org and www.kivunim.com, respectively) on their own initiative to get help in their career decision-making process. Only participants who filled out both the Career Decision Difficulties Questionnaire $(C D D Q)$ and the 
Career Decision Making Profiles (CDMP) questionnaire were included in the analyses. The third and fourth samples consisted of undergraduate students from American universities $(\mathrm{N}=915)$ and Chinese universities $(\mathrm{N}=929)$ who filled out the questionnaires in return for a report about their career decision-making adaptability.

Specifically, the US-Adult (US-A) sample included 804 individuals who entered the English version of the "Future Directions" website in 2011-2012, and reported coming from an English-speaking country (84.5\% from the United States, $8.1 \%$ from Canada, and $7.4 \%$ from other English-speaking countries). Because a vast majority of the participants in this group reported the US as their country of origin, we refer to this group as a US-Adult group. From this group 203 individuals were excluded from the analyses for one or more of the following reasons: (a) the duration of filling out one of the questionnaires was less than two minutes (which probably indicates that they did not fill out the questionnaire with proper attention) or more than half an hour, (b) they replied to one or more of the four validity items (see below) in a way that indicates questionable attention, or (c) they were under 18 or over 50 . Of the 601 remaining individuals in this group, 427 were female (71\%) and 174 were male (29\%), and their mean age was 30.60 ( $S D=8.81$ ). The second group, the Israeli sample, included 696 Israeli individuals who entered the Future Directions website during 2010-2012. Seventy-three individuals from this group were excluded from the analyses for one or more of the reasons listed above. Of the 623 remaining individuals in this group, 400 were female $(64.2 \%)$ and 223 were male $(35.8 \%)$, and their mean age was $25.06(S D=6.24)$.

The data of the third and fourth groups was collected between March 2013 and October 2013 as part of the study by Guan and his colleagues (2015). The data were collected from 12 universities in the United State and 12 universities in China (for further details see Guan et al., 2015). The students filled out a series of online questionnaires; in the current study we analyzed 
the $C D M P$ and the $C D D Q$ data. As a token of gratitude for participating in the study, the students later received feedback about the adaptability of their career decision-making profile.

Specifically, the US-Students (US-S) sample included 958 undergraduate students from American universities. From this group, 43 individuals were excluded from the analysis due to missing data in the questionnaires used in the study conducted by Guan et al., (2015) or due to questionable answers to the validity items. Of the remaining 915 individuals 466 were female $(50.9 \%)$ and 449 were male $(49.1 \%)$, and their mean age was $21.67(S D=1.94)$. Even though the age of the participants in this group overlaps that of the first group, in the current study we decided not to merge the two US groups. The reason for this is our underlying assumption that undergraduate students, most of whom have already decided on their major, are necessarily different from deliberating individuals who filled out the questionnaires to receive help as part of their career decision-making. Furthermore, while the US-Students sample consisted of undergraduate students, the age and education of the US-Adults sample suggests that most of the individuals in this group had already graduated from college and joined the work force.

The fourth group, the Chinese sample, included 979 undergraduate students from Chinese universities. From this group, 50 individuals were excluded from the analysis due to missing data in the questionnaires used in Guan et al.'s (2015) study or questionable answers to the validity items. Of the remaining 929 individuals, 520 (56\%) were female and 409 were male (43\%), and their mean age was $21.78(S D=1.44)$, similar to the US-Students sample.

\section{Instruments}

The Career Decision-Making Profiles questionnaire (CDMP, Gati et al., 2010). For the US-A sample and the Israeli sample we used the 39-item version of the CDMP, whereas for the US-S sample and the Chinese sample the original 36-item version was used. The CDMP is a multidimensional self-report questionnaire which assesses the individual along 12 dimensions 
(for the 39-item version) and 11 dimensions (for the 36-item version, which does not include the Using intuition dimension). Participants were asked to rate, on a 7-point Likert-type scale, the degree to which they agree with each statement (1 - do not agree at all, 7 - highly agree). The first item of the CDMP is a warmup item ("I am concerned about choosing a major or an occupation"), and two validity items are embedded in the questionnaire. The remaining items are statements that belong to one of the 12 (or 11) dimensions. Each of the statements represents one of the two poles that anchor each dimension (e.g., the item "I tend to postpone my career decision" represents the high pole of the procrastination scale). The score of each of the scales is defined as the mean score of the three items that represent it. Additionally, we computed a Career Decision Adaptability score (CDA) for each participant according to the mean score of the six scales that had been found to be adaptive (Gadassi et al., 2012; Gati \& Levin, 2012). Previous research by Gati et al. (2012) reported, based on two samples, a median Cronbach's $\alpha$ internal consistency reliability score of .81 and .82 for the 12 dimensions. These results were later supported in a stability study by Gati and Levin (2012), which assessed Cronbach's $\alpha$ internal consistency reliability for the $12 C D M P$ dimensions on three different occasions in the course of a one-year follow-up study, with a median reliability of $.86, .87$, and .84 , respectively. Within-subject analyses (across the 12 dimensions) revealed high two-week reliability (median .90), and high one-year stability (median .81). In the current study, the CDMP's median Cronbach $\alpha$ internal consistency reliability estimates were $.78, .82, .80$, and .71, for the US-A, Israeli, US-S, and Chinese samples, respectively.

The Career Decision-Making Difficulties Questionnaire (CDDQ, Gati et al., 1996). The 34-item version of the $C D D Q$ was used in the first and second samples, and the original 44-item version for the third and fourth samples (Gati \& Saka, 2001b). The $C D D Q$ is a multidimensional questionnaire which describes each individual's difficulties in term of the ten categories. In the 
US-A and the Israeli samples, each of the participants was asked to rate, on a 9-point Likert-type scale, the degree to which each statement describes them (1-does not describe me to 9-describes me well); in the US-S and Chinese samples, each of the participants was asked to do the same on a 7-point Likert-type scale. The score of the ten difficulty scales is defined as the mean of each of the items in the category, and the score of the three major clusters is defined as the mean of the difficulty categories in the cluster. Additionally, we computed a total $C D D Q$ score, which is defined as the mean score of the ten difficulty scales. In previous research on the $C D D Q$, its psychometric qualities were found to be satisfactory. Gati et al. (1996) found that the median Cronbach $\alpha$ reliability of the ten difficulty scales was .78 and .77 , in an Israeli and a US sample, respectively. This result was supported by additional research (Osipow \& Gati, 1998). Furthermore, Gati et al. (1996) found that the median test-retest reliability scores for the three major clusters and the total $C D D Q$ score were $.67, .74, .72$, and .80 , respectively. In the current study, the $C D D Q$ 's median Cronbach $\alpha$ reliabilities were $.82, .70, .90$, and .87 , for the US-A, Israeli, US-S, and Chinese samples, respectively.

\section{Procedure}

The participants in the first and second samples entered either the English or the Hebrew version of the "Future Directions" website on their own initiative (to get help in career decisionmaking), and filled out both the $C D D Q$ and the $C D M P$ in order to receive individualized feedback about the focuses of their difficulties and the way they make career decisions, respectively. The participants in the third and fourth samples were undergraduate students at universities in the US and China, who filled out the $C D D Q$ and the $C D M P$ as part of a study in which they were asked to fill out a series of questionnaires in exchange for personal feedback (Guan et al., 2015).

\section{Preliminary Analyses}


We computed each participant's mean score for each of the CDMP dimensions and $C D A$ score (based on the six dimensions with the more adaptive poles, as described in the Introduction). Next, we computed each participant's score on the ten difficulty categories, and then, based on them, the scores for the three major $C D D Q$ clusters and the total $C D D Q$ score. Because the scores on the $C D D Q$ for the US-S and the Chinese sample were rated on a 7-point Likert-type scale, we linearly transformed the mean of the ten difficulty scale scores of each participant to a 9-point scale. Based on the transformed scale scores, we computed the scores for the three major clusters of the $C D D Q$ and the total $C D D Q$ score. Then, we tested the order effect (i.e., filling out the $C D D Q$ before or after the $C D M P$ ) for the US-A and Israeli samples by comparing the respective mean scale scores. After the Bonferroni corrections for multiple comparisons, only one order effect remained (out of 54), indicating no order effect. We also carried out a between-genders comparison of the scores on the $C D M P$ and the $C D D Q$, which revealed that, even though we used large sample sizes $(601,623,915$, and 929), after the Bonferroni corrections for multiple comparisons only 12 of the 106 gender comparisons were statistically significant. Since only 13 (of the 160) order and gender differences remained, we report the analyses in the Results section across the two orders of administration and across gender.

\section{Results}

Table 1 presents the means (and $S D$ s) of the dimension scores in the CDMP as well as the Cronbach $\alpha$ internal consistency reliability estimates in each sample. It also presents the results of the ANOVA comparing the four samples and the effect size of the differences among them. Due to the large $N$, which produces statistical significance even for negligible differences, in the Results section we regard only differences of $\eta^{2} \geq .20$ as meaningful. The comparison of the mean $C D M P$ dimension scores among the four samples revealed negligible group differences, 
with only one noticeable difference $\left(\eta^{2}=.30\right)$ for the dimension of Desire to please others. In this dimension the Chinese sample was the highest $(M=4.63)$ whereas the US-A sample was the lowest $(M=2.51)$. The samples also differ in their $C D A$ score $\left(\eta^{2}=.20\right)$; the US-A sample had the highest $C D A$ score $(M=5.13)$ and the Chinese sample had the lowest $(M=3.86)$.

Table 2 presents the means (and $S D s$ ) of the three major cluster scores of the $C D D Q$ and the total $C D D Q$ score, as well as the Cronbach $\alpha$ internal-consistency reliability estimates in each of the samples. It also presents the results of the ANOVA comparing the four samples and the effect sizes of the differences among the four samples. The samples differed in one of the three major difficulty cluster scores - Inconsistent Information $\left(\eta^{2}=.24\right)$, where the Chinese sample was the highest $(M=5.24)$ and the Israeli sample was the lowest $(M=3.05)$.

\section{The Associations between the $C D M P$ and the $C D D Q$}

Table 3 presents the Pearson correlations between the $12 C D M P$ dimensions and the $C D A$ score, on the one hand, and the three major clusters and the total $C D D Q$ score, on the other. Due to the large $N$, which produces statistical significance even for negligible differences, in the Results section we regard only associations reflected in a correlation of $r \geq|.20|$ as worthy of attention, while correlations of $r \geq|.32|$ (reflecting at least ten percent of the common variance) are presented in bold. The difference between correlations regarded as negligible $(r<|.20|)$ and those reflecting a significant association $(r \geq|.32|)$ were all statistically significant.

The US-A sample. In all three $C D D Q$ clusters (Lack of Readiness, Lack of Information and Inconsistent Information) and the total $C D D Q$ score, mainly moderate to high correlations were observed between the $C D D Q$ and the $C D M P$ dimensions of Locus of control $(-.34 \leq r \leq-$ $.22)$, Procrastination $(.54 \leq r \leq .64)$, Speed of making the final decision $(-.61 \leq r \leq-.52)$, Dependence on others $(.35 \leq r \leq .49)$, and Desire to please others $(.23 \leq r \leq .33)$. The $C D A$ was strongly associated with all three clusters of the $C D D Q$ and the total $C D D Q$ score $(-.67 \leq r \leq-$ 
$.56)$.

The Israeli sample. Moderate to high correlations were observed between the clusters and the total $C D D Q$ score, on the one hand, and the CDMP dimensions of Locus of control $(-.38 \leq r$ $\leq-.28)$, Dependence on others $(.24 \leq r \leq .43)$ and Desire to please others $(.22 \leq \mathrm{r} \leq .43)$, on the other. A moderate to high association was observed between Lack of Readiness, Inconsistent Information, and the total $C D D Q$ score, on the one hand, and the $C D M P$ dimensions of Procrastination $(.24 \leq r \leq .4)$ and Speed of making the final decision $(-.49 \leq r \leq-.28)$ on the other. The $C D A$ was strongly associated with all three clusters of the $C D D Q$ and the total $C D D Q$ score $(-.62 \leq r \leq-.35)$.

The US-S sample. Moderate to high correlations were observed between the clusters and the total $C D D Q$ score, on the one hand, and the CDMP dimensions of Information gathering ($.40 \leq r \leq-.27)$, Locus of control $(-.40 \leq r \leq-.30)$, Procrastination $(.39 \leq r \leq .52)$, Speed of making the final decision $(-.38 \leq r \leq-.24)$, Dependence on others $(.41 \leq r \leq .48)$ and Desire to please others $(.29 \leq \mathrm{r} \leq .34)$, on the other. A moderate to high association was observed between Lack of Information, Inconsistent Information and the total $C D D Q$ score, on the one hand, and Aspiration for an ideal occupation $(-.28 \leq r \leq-.21)$, on the other. The $C D A$ was strongly associated with all three clusters of the $C D D Q$ and the total $C D D Q$ score $(-.62 \leq r \leq-.53)$.

The Chinese sample. Moderate to high correlations were observed between the clusters and the total $C D D Q$ score, on the one hand, and the $C D M P$ dimensions of Information gathering $(-.33 \leq r \leq-.25)$, Locus of control $(-.39 \leq r \leq-.22)$, Procrastination $(.29 \leq r \leq .42)$ and Consulting with others $(-.34 \leq r \leq-.25)$, on the other. A moderate to high association was found between Lack of Readiness, Lack of Information, and the total CDDQ score, on the one hand, and Dependence on others $(.29 \leq r \leq .39)$, on the other. The $C D A$ was highly associated with all three clusters of the $C D D Q$ and the total $C D D Q$ score $(-.49 \leq r \leq-.31)$. 


\section{Regression Analyses}

To further investigate the association between the $C D M P$ and the $C D D Q$, we carried out a two-step hierarchical multiple regression analyses in each of the samples, with the Lack of Readiness major cluster of the $C D D Q$ as the dependent variable. In step one, the $C D A$ was entered in the analysis, and at step two the 12 dimensions of the $C D M P$ were entered as the additional independent variables. We repeated this analysis with the two other major $C D D Q$ clusters (Lack of Information and Inconsistent Information) and the total CDDQ score as the dependent variables. Only variables whose inclusion in the regression analyses increased the $R^{2}$ by at least .03 were regarded as significant predictors.

Lack of Readiness. The results of the multiple regression analysis revealed that for all samples the CDMP significantly predicted difficulties related to Lack of Readiness (Adjusted $R^{2}$ $=.42, .38, .29$, and .26 , for the US-A, Israeli, US-S, and Chinese samples, respectively; all were statistically significant $-F(1,600)=442.70, F(1,621)=383.92, F(1,914)=371.72, F(2,928)$ $=167.31$, respectively. The $C D A$ score, that was entered first, significantly predicted difficulties related to Lack of Readiness in all four samples $\left(\beta=-.65,-.62,-.54\right.$ and -.42 , respectively; $\Delta R^{2}$ $=.42, .38, .29$, and .24 , respectively). Only in the Chinese sample an additional predictor emerged - Consulting with others added to the prediction of difficulties related to Lack of Readiness $(\beta=$ -.18 and $\left.\Delta R^{2}=.03\right)$

Lack of Information. The results of the multiple regression analysis revealed that for all samples the CDMP significantly predicted difficulties involving Lack of Information (Adjusted $R^{2}=.36, .12, .34$, and .21 for the US-A, Israeli, US-S, and Chinese samples, respectively; all were statistically significant $-F(2,600)=167.00, F(1,621)=88.13, F(2,914)=236.18, F$ $(2,928)=121.10$, respectively. These $R^{2}$ s are smaller than those for Lack of Readiness. The CDA score, which was entered first, significantly predicted difficulties involving Lack of Information 
$\left(\beta=-.30,-.35,-.52\right.$ and -.43 , respectively; $\Delta R^{2}=.31, .12, .31$ and .17 , respectively $)$

Additionally, in three of the samples an additional predictor emerged: Procrastination for the US-A sample $\left(\beta=.34\right.$ and $\left.\Delta R^{2}=.05\right)$, Aspiration for an ideal occupation for the US-S sample $(\beta$ $=-.17$ and $\left.\Delta R^{2}=.03\right)$, and Information processing for the Chinese sample $\left(\beta=-.21\right.$ and $\Delta R^{2}$ $=.04)$.

Inconsistent Information. The results of the multiple regression analysis revealed that for all samples the CDMP significantly predicted difficulties involving Inconsistent Information (Adjusted $R^{2}=.37, .24, .28$, and .10 for the US-A, Israeli, US-S, and Chinese samples, respectively; all were statistically significant $-F(2,600)=175.16, F(2,621)=99.62, F(1,914)=$ 364.97, $F(1,928)=99.40$, respectively. The $C D A$ score, which was entered first, significantly predicted difficulties involving Inconsistent Information $(\beta=-.39,-.30,-.53$ and -.31 , respectively; $\Delta R^{2}=.34, .21, .29$ and .10 , respectively). Moreover, in two of the samples an additional predictor emerged: Procrastination for the US-A sample $\left(\beta=.26\right.$ and $\left.\Delta R^{2}=.03\right)$ and Desire to please others for the Israeli sample $\left(\beta=.24\right.$ and $\left.\Delta R^{2}=.03\right)$.

The total $C D D Q$ score. The multiple regression analysis revealed that for all of the samples the $C D M P$ significantly predicted the total $C D D Q$ score $\left(\right.$ Adjusted $R^{2}=.48, .33, .38$, and .25 for the US-A, Israeli, US-S, and Chinese samples, respectively; all were statistically significant $-F(2,600)=283.03, F(1,621)=310.43, F(1,914)=557.25, F(2,928)=156.78$, respectively. The $C D A$ score, that was entered first, was the best predictor of the total $C D D Q$ score in all four samples $\left(\beta=-.44,-.58,-.62\right.$ and -.41 , respectively; $\Delta R^{2}=.45, .33, .38$ and .23 , respectively). Moreover, in two of the samples an additional predictor emerged: Procrastination for the US-A sample $\left(\beta=.31\right.$ and $\left.\Delta R^{2}=.04\right)$ and Consulting with others for the Chinese sample $\left(\beta=-.18\right.$ and $\left.\Delta R^{2}=.03\right)$.

\section{Discussion}


The present study investigated the associations between career decision-making profiles $(C D M P)$ and career decision-making difficulties $(C D D Q)$ in four large samples - US-Adults, Israeli, US-Student, and Chinese. We focused on the associations between the 12 CDMP dimension scores and the index of career decision adaptability $(C D A)$ derived from it, on the one hand, and the total $C D D Q$ score and the three $C D D Q$ major cluster scores (Lack of Readiness, Lack of Information, and Inconsistent Information), on the other.

Overall, the pattern of associations between the $C D M P$ and the $C D D Q$ were very similar across all four samples. First, the associations were in the same direction - positive in all four samples or negative in all four samples. Second, an internal Locus of control, less Procrastination, faster Speed of making the final decision, and less Dependence on others were associated with fewer difficulties in all the samples. In addition, a more comprehensive Information gathering (excluding only the US-Adults sample) and less Desire to please others (excluding only the Chinese sample) were also associated with fewer difficulties in three samples. Most interestingly, these dimensions of the CDMP were all found to be more adaptive in career decision-making and are included in the $C D A$ index. Third, a strong association was found between a higher $C D A$ index and fewer career decision-making difficulties in all four samples. The regression analyses, which tested whether there are dimensions that predict difficulties beyond the $C D A$, revealed that across samples and difficulty clusters the $C D A$ captured most of the variance in the prediction of career decision-making difficulties with no more than one other dimension as an additional predictor, indicating the importance of career decision adaptability for predicting fewer difficulties. Furthermore, these associations are compatible with the results of Gadassi et al. (2012), who found that these dimensions of the $C D M P$ were associated with fewer emotional and personality-related career decision-making difficulties. 
US samples. An unpredicted finding is that, overall, the $C D A$ accounted for career decision-making difficulties better in the US samples (both adults and students) than in the Israeli and Chinese samples. In addition, a few unique associations emerged in the US samples. Firstly, only in the US samples we found a correlation between greater Speed of making final decision and fewer difficulties involving Lack of Information. Perhaps it takes a long time to make a decision in American society, with its abundance of information and easy access to it, because individuals' continue to feel that they have not collected all the relevant information. Future research should explore this possibility. Secondly, in the US-Adults sample Procrastination contributed to the prediction of the overall $C D D Q$ score beyond the $C D A$, although it is one of six dimensions that define the CDA index. This implies that Procrastination plays a more important role than the other adaptive dimensions in career decision-making difficulties for this sample. Thirdly, more comprehensive Information gathering was not found to be correlated with the $C D D Q$ in the US-Adults sample; interestingly, however, it was associated with the $C D D Q$ in the US-Students sample. This discrepancy can be attributed, at least partially, to the younger age of the US-Student sample; further research should determine the reasons for it. Finally, only in the US-Students sample we found correlations between Aspiration for an ideal occupation and difficulties related to Lack of Information and Inconsistent Information. Perhaps aspiring for an ideal occupation makes you feel that you do not have enough information.

Israeli sample. One unique result emerged in the Israeli sample - Desire to please others was as a predictor for difficulties involving Inconsistent Information, in addition to the CDA index. This result is especially interesting since Desire to please others is one of the dimensions included in the $C D A$ index. This indicates that the $C D A$ underweights the negative contribution of Desire to please others to adaptive career decision making for Israelis. This association is caused mainly by the significant association between Desire to please others and difficulties 
involving external conflicts, which is included in Inconsistent Information. In Israeli society, which is a partly individualistic and partly collectivistic society, individuals who are high on Desire to please others, may feel that the pressure of significant others increases their sense of conflict; future research should try to account for this result.

Chinese sample. A few unique results emerged in the Chinese sample. First, only in this sample were the correlations between Consulting with others and career decision-making difficulties negative - unlike the results for the other samples, for Chinese students consulting with others actually reduces difficulty. Specifically, Consulting with others emerged as a predictor for fewer difficulties involving Lack of Readiness and the total $C D D Q$ score (in addition to the $C D A$ index). This may be explained by the collectivistic orientation of the Chinese society (Yi \& Park, 2003), where one tends to forgo one's own goals for the sake of the goals of the group, and see one's own fate as intertwined with that of the group (Triandis, 1990). Consultation with others may play a role not only in giving the individual information about what is expected of him or her, but also telling the individual what is the "right" decision to make, thus reducing distress and difficulty.

Second, in addition to the $C D A$ index, more analytic Information processing emerged as a predictor for fewer difficulties involving Lack of Information. This finding is especially interesting because more holistic Information Processing is generally attributed to Chinese society (Nisbett \& Miyamoto, 2005). Third, only for the Chinese sample was Desire to please others uncorrelated with the difficulties that arise during decision-making-Lack of Information and Inconsistent Information. Western cultures usually promote individualism (Mau, 2004), and thus in these cultures an individual with a high desire to please others will feel conflicted between the desire to please significant others in his or her life, and the cultural norm that encourages making a personally satisfying decision. This conflict can lead to difficulties in 
decision-making. It seems that, unlike Western cultures, Chinese society avoids this conflict because the individual is expected to feel a desire to please others when making a career decision.

In summary, the present research indicates that in cross-cultural research focusing only on the six dimensions that were found to be adaptive in career decision-making, and hence included in the $C D A$, is highly informative in accounting for the difficulties individuals face in career decision-making. Although some unique associations did emerge, additional research is needed before deciding that the $C D A$ should be adapted to better fit a variety of cultures by adding other dimensions to it. Independent of this question, the result supports the definition and validity of the $C D A$ index, indicating that in most situations using the six adaptive $C D M P$ dimensions, rather than all 12, provides an adequate assessment.

\section{Limitations}

Before we discuss the implications of the current study, its limitations have to be acknowledged. First, in the US-Adults sample the participants were older (mean age $=31$ ) than the typical age for making career decisions involving post-secondary-school training or a college major. Thus, further research should determine the characteristics of this sample and the reasons why these individuals chose to use an Internet-based career self-help instrument. Second, two of our samples (US-Students and Chinese) included undergraduate students (mean years in college $=2.91$ and 2.84 , respectively) who had already advanced in their career decision-making to some extent, and so future research is needed for the associations between the CDMP and the $C D D Q$ to be generalizable to a broader group of young adults deliberating about their future careers. Third, further research should directly test cultural explanations for the differences found among the samples. Last, the current study focused on only three cultures, and thus further research should test which results can be generalized to other cultures. 


\section{Implications}

In light of the differences among the samples, the similarities in the pattern of results are especially interesting. First, as hypothesized, across the three cultures we found that the more adaptive one's career decision-making profile the fewer difficulties one has in making a career decision. This suggests that career counselors can construct interventions by focusing on the six adaptive dimensions, to help individuals adopt more adaptive ways of making career decisions (Gadassi et al., 2013). Doing so will reduce career decision-making difficulties, thus helping the individual advance towards making the decision. Second, the present research supports the validity of the construct of Career Decision-making Adaptability. The fact that the highest correlations between the $C D M P$ and the $C D D Q$ were found in the six adaptive dimensions, and not in the other six, further supports the definition of the CDA index (Gati \& Levin, 2012). However, it is important to assess whether it is necessary to change the operationalization of career decision-making adaptability (i.e., what dimensions it includes) for different cultures, based on the association between career decision-making profiles and difficulties, as well as additional criteria. Third, although the associations between career decision-making profiles and difficulties were largely replicated across different cultures, certain differences did emerge. This result indicates that it may be necessary to reevaluate which dimensions of the career decisionmaking profiles are indeed adaptive in many cultures and which are relevant only for Western cultures. 


\section{References}

Blustein, D. L., \& Ellis, M. V. (2000). The cultural context of career assessment. Journal of Career Assessment, 8, 379-390. doi:10.1177/106907270000800407

Campbell, R. E., \& Cellini, J. V. (1981). A diagnostic taxonomy of adult career problems. Journal of Vocational Behavior, 19, 175-190. doi:10.1016/0001-8791(81)90057-9

Clark, B. R. (1986). The higher education system: Academic organization in cross-national perspective. California: University of California Press.

Creed, P. A., \& Yin, W. O. (2006). Reliability and validity of a Chinese version of the career decision-making difficulties questionnaire. International Journal for Educational and Vocational Guidance, 6, 47-63. doi: 10.1007/s10775-006-0003-3

Di Fabio, A., \& Palazzeschi, L. (2009). Emotional intelligence, personality traits and career decision difficulties. International Journal for Educational and Vocational Guidance, 9, 135146. doi: 10.1007/s10775-009-9162-3

Fouad, N., Cotter, W. E., \& Kantamneni, N. (2009). The effectiveness of a career decisionmaking course. Journal of Career Assessment, 17, 338-347. doi: $10.1177 / 1069072708330678$

Gadassi, R., Gati, I., \& Dayan, A. (2012). The adaptability of career decision-making profiles. Journal of Counseling Psychology, 59, 612-622. doi:10.1037/a0029155

Gadassi, R., Gati, I., \& Wagman-Rolnick, H. (2013). The adaptability of career decision-making profiles: Associations with self-efficacy, emotional difficulties, and decision status. Journal of Career Development, 40, 490-507. doi: 10.1177/0894845312470027

Gati, I. (1986). Making career decisions: A sequential elimination approach. Journal of Counseling Psychology, 33, 408-417. doi: 10.1037/0022-0167.33.4.408

Gati, I., Gadassi, R., \& Mashiah-Cohen, R. (2012). Career decision-making profiles vs. styles: 
Convergent and incremental validity. Journal of Vocational Behavior, 81, 2-16. doi:10.1016/j.jvb.2012.03.004

Gati, I., Krausz, M., \& Osipow, S. H. (1996). A taxonomy of difficulties in career decisionmaking. Journal of Counseling Psychology, 43, 510-526. doi:10.1037/0022-0167.43.4.510

Gati, I., Landman, S., Davidovitch, S., Asulin-Peretz, L., \& Gadassi, R. (2010). From career decision-making styles to career decision-making profiles: A multidimensional approach. Journal of Vocational Behavior, 76, 277-291. doi: 10.1016/j.jvb.2009.11.001

Gati, I., \& Levin, N. (2012). The stability and structure of career decision-making profiles: A one-year follow-up. Journal of Career Assessment. 20, 4, 390-403. doi:

$10.1177 / 1069072712448892$

Gati, I., Osipow, S. H., Krausz, M., \& Saka, N. (2000). Validity of the career decision-making difficulties questionnaire: Counselee versus career counselor perceptions. Journal of Vocational Behavior, 56, 99-113. doi:10.1006/jvbe.1999.1710

Gati, I., \& Saka, N. (2001a). High school students' career related decision-making difficulties. Journal of Counseling and Development, 79, 331-340. doi:10.1002/j.15566676.2001.tb01978.x

Gati, I., \& Saka, N. (2001b). Internet-based versus paper-and-pencil assessment: Measuring career decision-making difficulties. Journal of Career Assessment, 9, 397-416. doi:10.1177/106907270100900406

Gillie, S., \& Gillie-Isenhour, M. (2003). The educational, social and economic value of informed and considered career decisions. Alexandria, VA: America's Careers Resource Network Association.

Guan, Y., Bond, M., Levin, N., Chen, S., Guo, Y., Deng, H., Xu, J., Zhou, X., Ji, Y., Li, C., Han, X., \& Chen, P. (2015). Differences in career decision-making profiles between 
American and Chinese university students: The relative strength of mediating mechanisms across cultures. Submitted for publication.

Harren, V. A. (1979). A model of career decision making for college students. Journal of Vocational Behavior, 14, 119-133. doi:10.1016/0001-8791(79)90065-4

Hui, C. H., \& Triandis, H. C. (1986). Individualism-collectivism: A study of cross cultural researchers. Journal of Cross-Cultural Psychology, 17, 225-248. doi:

$10.1177 / 0022002186017002006$

Hui, C. H., \& Villareal, M. R. (1989). Individualism-collectivism and psychological needs: Their relationships in two cultures. Journal of Cross-Cultural Psychology, 20, 310-323. doi: $10.1177 / 0022022189203005$

Katz, M. (1966). A model of guidance for career decision-making. Vocational Guidance Quarterly, 15, 2-10. doi:10.1002/j.2164-585X.1966.tb01148.x

Kelly, K. R., \& Lee, W.C. (2002). Mapping the domain of career decision problems. Journal of Vocational Behavior, 61, 302-326. doi:10.1006/jvbe.2001.1858

Kleiman, T., Gati, I., Peterson, G., Sampson, J., Reardon, R., \& Lenz, J. (2004). Dysfunctional thinking and difficulties in career decision making. Journal of Career Assessment, 12, 312331. doi:10.1177/1069072704266673

Lancaster, P. L., Rudolph, C., Perkins, S., \& Patten, T. (1999). Difficulties in career decision making: A study of the reliability and validity of the career decision difficulties questionnaire. Journal of Career Assessment, 4, 393-413. doi:10.1177/106907279900700405

Lease, S. H. (2004). Effect of locus of control, work knowledge, and mentoring on career decision-making difficulties: Testing the role of race and academic institution. Journal of Career Assessment, 12, 239-254. doi:10.1177/1069072703261537

Lipshits-Braziler, Y., Gati, I., \& Tatar, M. (2015). Strategies for coping with career indecision. 
Journal of Career Assessment. doi: 10.1177/1069072714566795

Mau, W. C. (2000). Cultural differences in career decision-making styles and self-efficacy. Journal of Vocational Behavior, 57, 365-378. doi:10.1006/jvbe.1999.1745

Mau, W. C. (2001). Assessing career decision-making difficulties: A cross-cultural study. Journal of Career Assessment, 9, 353-364. doi:10.1177/106907270100900403

Mau, W. C. (2004). Cultural dimensions of career decision-making difficulties. The Career Development Quarterly, 53, 67-77. doi:10.1002/j.2161-0045.2004.tb00656.x

Miller, R. G. (1971). Development of a taxonomy of human performance: Design of a systems task vocabulary (Tech. Rep. A1R-72 6/2035-3/71-TRJI). Silver Spring, MD: AIR.

Nisbett, R. E., \& Miyamoto, Y. (2005). The influence of culture: Holistic versus analytic perception. Trends in Cognitive Sciences, 9, 467-473. doi:10.1016/j.tics.2005.08.004

Osipow, S. H. (1999). Assessing career indecision. Journal of Vocational Behavior, 55, 147154. doi:10.1006/jvbe.1999.1704

Osipow, S. H., \& Gati, I. (1998). Construct and concurrent validity of the career decision-making difficulties questionnaire. Journal of Career Assessment, 6, 347-364. doi:10.1177/106907279800600305

Perdrix, S., Stauffer, S., Masdonati, J., Massoudi, K., \& Rossier, J. (2012). Effectiveness of career counseling: A one-year follow-up. Journal of Vocational Behavior, 80, 565-578. doi:10.1016/j.jvb.2011.08.011

Pitz, G. F., \& Harren, V. A. (1980). An analysis of career decision making from the point of view of information processing and decision theory. Journal of Vocational Behavior, 16, 320-346. doi:10.1016/0001-8791(80)90059-7

Savickas, M. L. (2005). The theory and practice of career construction. In S.D. Brown, R.W. Lent (Eds.), Career development and counselling: Putting theory and research to work (pp. 
42-70). Wiley, Hoboken, NJ.

Sidiropoulou-Dimakakou, D., Mylonas, K., Argyropoulou, K., \& Tampouri, S. (2012). Career decision-making difficulties, dysfunctional thinking and generalized self-efficacy of university students in Greece. World Journal of Education, 2, 117-130. doi:10.5430/wje.v2n1p117

Tian, L., Guan, Y., Chen, S. X., Levin, N., Cai, Z., Chen, P., Zhu, C., Fu, R., Wang, Y., \& Zhang, S. (2014). Predictive validity of career decision-making profiles over time among Chinese college students. Journal of Career Development. 41, 4, 282-300. doi: $10.1177 / 0894845313495590$

Tien, H. L. S. (2005). The validation of the career decision making difficulties scale in a Chinese culture. Journal of Career Assessment, 13, 114-127. doi: 10.1177/1069072704270327

Tinsley, H. E. A. (1992). Career decision making and career indecision. Journal of Vocational Behavior, 41, 209-211. doi: 10.1016/0001-8791(92)90022-R

Triandis, H. C. (1990). Cross-cultural studies of individualism and collectivism. In J.J. Berman (Ed.), Nebraska Symposium on motivation, 1989: cross-cultural perspectives (Vol. 37, pp. 41-133). Lincoln: University of Nebraska Press.

Vahedi, S., Farrokhi, F., Mahdavi, A., \& Moradi, S. (2012). Exploratory and confirmatory factor analysis of the career decision-making difficulties questionnaire. Iranian Journal of Psychiatry, 7, 74-81.

Vertsberger, D., \& Gati, I. (2015). Career decision-making difficulties and help-seeking among Israeli young adults. Submitted for publication.

Yi, J. S., \& Park, S. (2003). Cross-cultural differences in decision-making styles: A study of college students in five countries. Social Behavior and Personality, 31, 35-47. doi: $10.2224 /$ sbp.2003.31.1.35 
$\underline{\text { Table } 1}$

Means, Standard Deviations, C $\alpha$ Reliability Estimates of the CDMP Scores, and the Results of the ANOVA Comparing the Four Samples

\begin{tabular}{|c|c|c|c|c|c|c|c|c|c|c|c|c|c|c|}
\hline \multirow[b]{2}{*}{ Scale } & \multicolumn{3}{|c|}{ US-A $(\mathrm{N}=601)$} & \multicolumn{3}{|c|}{ Israel $(\mathrm{N}=623)$} & \multicolumn{3}{|c|}{ US-S $(\mathrm{N}=915)$} & \multicolumn{3}{|c|}{ China $(\mathrm{N}=929)$} & \multirow[b]{2}{*}{$F(3,3067)$} & \multirow[b]{2}{*}{ Partial $\eta^{2}$} \\
\hline & Mean & $\mathrm{SD}$ & $C_{\alpha}$ & Mean & $\mathrm{SD}$ & $C_{\alpha}$ & Mean & SD & $\overline{C_{\alpha}}$ & Mean & $\mathrm{SD}$ & $C_{\alpha}$ & & \\
\hline$I G$ & 5.42 & 1.12 & .74 & 4.59 & 1.59 & .80 & 5.24 & 1.42 & .70 & 4.22 & 1.23 & .70 & 137.64 & .12 \\
\hline IP & 4.57 & 1.45 & .62 & 4.50 & 1.43 & .77 & 5.54 & 0.96 & .76 & 5.10 & 0.96 & .71 & 129.41 & .11 \\
\hline$L C$ & 5.37 & 1.29 & .76 & 5.37 & 1.41 & .79 & 4.81 & 1.39 & .78 & 4.18 & 1.16 & .70 & 147.41 & .13 \\
\hline EI & 5.09 & 1.23 & .81 & 5.23 & 1.34 & .83 & 5.39 & 0.98 & .79 & 4.58 & 0.96 & .70 & 89.40 & .08 \\
\hline$P R$ & 3.55 & 1.86 & .92 & 4.34 & 1.79 & .88 & 3.89 & 1.58 & .86 & 4.12 & 1.25 & .86 & 28.54 & .03 \\
\hline$S P$ & 4.51 & 1.78 & .89 & 2.95 & 1.67 & .86 & 3.35 & 1.32 & .80 & 3.32 & 1.10 & .71 & 137.17 & .12 \\
\hline $\mathrm{CO}$ & 5.05 & 1.37 & .73 & 5.33 & 1.39 & .79 & 4.28 & 1.45 & .82 & 4.74 & 1.26 & .80 & 83.23 & .08 \\
\hline$D O$ & 2.47 & 1.28 & .75 & 2.76 & 1.61 & .82 & 3.07 & 1.35 & .78 & 3.81 & 1.13 & .70 & 146.75 & .13 \\
\hline$D P$ & $2.51^{\mathrm{bd}}$ & 1.31 & .80 & $2.63^{\mathrm{ce}}$ & 1.44 & .87 & $3.27^{\mathrm{abc}}$ & 1.50 & .86 & $4.63^{\text {ade }}$ & 1.03 & .70 & 433.26 & .30 \\
\hline AI & 4.58 & 1.65 & .86 & 4.82 & 1.44 & .77 & 5.22 & 1.22 & .86 & 4.52 & 1.15 & .78 & 49.77 & .05 \\
\hline WC & 4.37 & 1.48 & .86 & 3.90 & 1.52 & .88 & 4.80 & 1.33 & .87 & 5.01 & 1.00 & .78 & 101.70 & .09 \\
\hline IN & 4.26 & 1.26 & .75 & 4.40 & 1.45 & .86 & & & 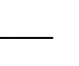 & & & 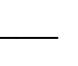 & 3.29 & .00 \\
\hline$C D A$ & $5.13^{\text {bce }}$ & 0.94 & .71 & $4.53^{\mathrm{de}}$ & 0.99 & .68 & $4.53^{\mathrm{ab}}$ & 0.96 & .76 & $3.86^{\mathrm{acd}}$ & 0.74 & .71 & 251.99 & .20 \\
\hline
\end{tabular}

Notes: Abbreviations used: $I G=$ information gathering; IP, information processing; LC, locus of control; EI, effort invested; PR, procrastination; SP, speed of making the final decision; $\mathrm{CO}$, consultation with others; $D O$, dependence on others; DP, desire to please others; AI, aspiration for an ideal occupation; WC, willingness to compromise; IN, using intuition; CDA, career decision-making adaptability. Dimensions included in the $C D A$ are presented in italics.

Means with a similar superscript are statistically different (Tukey post-hoc test, $p<.05$ ); partial $\eta^{2} \geq .20$ is presented in bold. 
Table 2

Means, Standard Deviations, $\mathrm{C} \alpha$ Reliability Estimates of the Cluster $C D D Q$ Scores, and the Results of the ANOVA Comparing the Four Samples

\begin{tabular}{|c|c|c|c|c|c|c|c|c|c|c|c|c|c|c|}
\hline & \multicolumn{3}{|c|}{ US-A $(\mathrm{N}=601)$} & \multicolumn{3}{|c|}{ Israel $(\mathrm{N}=623)$} & \multicolumn{3}{|c|}{ US-S $(\mathrm{N}=915)$} & \multicolumn{3}{|c|}{ China $(\mathrm{N}=929)$} & \multirow[b]{2}{*}{$F(3,3067)$} & \multirow[b]{2}{*}{ Partial $\eta^{2}$} \\
\hline & Mean & $\mathrm{SD}$ & $C_{\alpha}$ & Mean & $\mathrm{SD}$ & $C_{\alpha}$ & Mean & SD & $\overline{C_{\alpha}}$ & Mean & $\mathrm{SD}$ & $\overline{C_{\alpha}}$ & & \\
\hline LR & 3.80 & 1.13 & .66 & 4.44 & 1.20 & .70 & 5.09 & 1.31 & .78 & 5.06 & 1.09 & .71 & 186.54 & .15 \\
\hline LI & 3.70 & 2.12 & .95 & 5.25 & 1.66 & .85 & 4.16 & 1.92 & .97 & 5.02 & 1.37 & .95 & 115.35 & .10 \\
\hline II & $3.05^{\text {bce }}$ & 1.64 & .89 & $3.87^{\mathrm{de}}$ & 1.40 & .74 & $3.89^{\mathrm{ab}}$ & 1.61 & .93 & $5.24^{\mathrm{acd}}$ & 1.04 & .89 & 318.95 & .24 \\
\hline CDDQ & 3.54 & 1.47 & .93 & 4.59 & 1.13 & .86 & 4.36 & 1.46 & .92 & 5.10 & 1.01 & .87 & 186.95 & .16 \\
\hline
\end{tabular}

Notes: Abbreviations used: LR, lack of readiness; LI, lack of information; II, inconsistent information; CDDQ, the total CDDQ score. Means with a similar superscript are statistically different (Tukey post-hoc test, $p<.05$ ); partial $\eta^{2} \geq .20$ is presented in bold. 


\section{$\underline{\text { Table } 3}$}

The Pearson Correlations between the CDMP and the $C D D Q$ : US-A sample $(\mathrm{N}=601)$, Israeli sample $(\mathrm{N}=623)$, US-S sample (N=915), and Chinese sample $(\mathrm{N}=929)$

\begin{tabular}{|c|c|c|c|c|c|c|c|c|c|c|c|c|c|c|c|c|}
\hline \multirow{3}{*}{$\begin{array}{c}\text { CDMP } \\
\text { dimensions }\end{array}$} & \multicolumn{12}{|c|}{ CDDQ clusters } & & & & \\
\hline & \multicolumn{4}{|c|}{ Lack of Readiness } & \multicolumn{4}{|c|}{ Lack of Information } & \multicolumn{4}{|c|}{ Inconsistent Information } & \multicolumn{4}{|c|}{ The total $C D D Q$ score } \\
\hline & US-A & IS & US-S & $\mathrm{CH}$ & US-A & IS & US-S & $\mathrm{CH}$ & US-A & IS & US-S & $\mathrm{CH}$ & US-A & IS & US-S & $\mathrm{CH}$ \\
\hline$I G$ & & & -.27 & -.27 & & -.23 & -.38 & -.32 & & & -.40 & -.25 & & -.27 & -.40 &.-33 \\
\hline IP & & & & & & & -.21 & & & & & & & & & \\
\hline$L C$ & -.34 & -.34 & -.30 & -.39 & -.22 & -.28 & -.36 & -.29 & -.32 & -.30 & -.39 & -.22 & -.31 & -.38 & -.40 & -.35 \\
\hline EI & & & & & & & -.20 & & & & & & & & & \\
\hline$P R$ & .54 & .45 & .48 & .41 & .57 & & .50 & .36 & .55 & .24 & .39 & .29 & .64 & .35 & .52 & .42 \\
\hline$S P$ & -.57 & -.49 & -.38 & & -.52 & & -.29 & & -.53 & -.28 & -.24 & & -.61 & -.37 & -.34 & -.21 \\
\hline $\mathrm{CO}$ & & & & -.34 & & & & -.26 & & & & -.25 & & & & -.33 \\
\hline$D O$ & .49 & .43 & .41 & .39 & .35 & .24 & .43 & .29 & .36 & .30 & .42 & & .43 & .39 & .48 & .34 \\
\hline$D P$ & .33 & .39 & .32 & .20 & .23 & .22 & .29 & & .28 & .43 & .32 & & .30 & .42 & .34 & \\
\hline AI & & & & .20 & & & -.28 & & & & -.21 & & & & -.24 & \\
\hline WC & & & & & .20 & & & & & & & & .20 & & & \\
\hline IN & & & - & - & & & - & - & & & - & -- & & & - - & - \\
\hline$C D A$ & -.65 & -.62 & -.54 & -.49 & -.56 & -.35 & -.56 & -.41 & -.58 & -.46 & -.53 & -.31 & -.67 & -.58 & -.62 & -.47 \\
\hline
\end{tabular}

Notes: IG, information gathering; IP, information processing; LC, locus of control; EI, effort invested; PR, procrastination; SP, speed of making the final decision; CO, consultation with others; DO, dependence on others; DP, desire to please others; AI, aspiration for an ideal occupation; WC, willingness to compromise; IN, using intuition; $C D A$, career decision-making adaptability (scales in italics define the $C D A$ ).

Correlations above .32 are presented in bold 